\title{
Efeito antioxidante da buspirona no modelo de epilepsia induzida por pilocarpina
}

\author{
Antioxidant effect of buspirone in epilepsy model induced by pilocarpine \\ Paula Benvindo Ferreira1, Antônia Amanda Cardoso de Almeida², Rivelilson Mendes de Freitas1,2
}

1 Curso de graduação em Farmácia do Departamento de Bioquímica e Farmacologia da Universidade Federal do Piauí (UFPI), Teresina, Piauí.

${ }_{2}$ Programa de Pós-graduação em Ciências Farmacêuticas do Centro de Ciências da Saúde da UFPI, Teresina, Piauí.

Recebido: 23/4/2012 - Aceito: 29/8/2012

\begin{abstract}
Resumo
Contexto: Crises epilépticas induzidas por pilocarpina podem produzir alterações histopatológicas em muitas regiões cerebrais como consequência da produção excessiva de radicais livres. Objetivo: O objetivo do presente estudo foi avaliar o efeito antioxidante da buspirona no modelo de epilepsia induzida por pilocarpina. Material e métodos: Quarenta e oito animais foram divididos em quatro grupos. O primeiro grupo foi tratado com solução salina $0,9 \%$ (Controle). O segundo grupo foi tratado com pilocarpina $400 \mathrm{mg} / \mathrm{kg}$ (P400). Por sua vez, o terceiro grupo foi tratado com buspirona $5 \mathrm{mg} / \mathrm{kg}$ (BUSP) durante 14 dias consecutivos. Já os animais do quarto grupo foram tratados com buspirona durante 14 dias consecutivos, e, 30 minutos após a última administração dela, os camundongos receberam P400 (BUSP + P400). Resultados: Durante o período do tratamento não se observaram sinais de toxicidade e nenhuma morte entre os animais tratados com buspirona. Em nosso estudo o grupo tratado com P400 demonstrou um aumento significativo da produção de nitrito e nos níveis de peroxidação lipídica após as crises epilépticas. Por outro lado, no hipocampo dos animais que receberam o pré-tratamento com buspirona e após 30 minutos receberam P400, foi observada redução significativa nos níveis de peroxidação lipídica (65\%) e nitrito (85\%), bem como aumento na atividade da enzima superóxido dismutase. Conclusão: O pré-tratamento com BUSP aumentou a latência para primeira crise epiléptica e diminuiu a taxa de mortalidade e o número de animais que apresentaram crise epiléptica e que progridem para o estado de mal epiléptico. Além disso, apresentou efeitos anticonvulsivantes associados com a redução do estresse oxidativo hipocampal no modelo de epilepsia induzida por pilocarpina.
\end{abstract}

Ferreira PB, et al. / Rev Psiq Clín. 2012;39(5):153-6

Palavras-chave: Buspirona, convulsões, estresse oxidativo, hipocampo, pilocarpina.

\begin{abstract}
Background: Pilocarpine-induced seizures can cause pathological changes in many brain regions as a result of excessive production of free radicals. Objective: The objective of this study was to evaluate the antioxidant effect of buspirone in the epilepsy model induced by pilocarpine. Material and methods: Forty-eight animals were divided into four groups. The first group was treated with saline $0.9 \%$ (control); the second group received pilocarpine $400 \mathrm{mg} / \mathrm{kg}$ (P400); the third group was treated with buspirone $5 \mathrm{mg} / \mathrm{kg}$ (BUSP) for 14 consecutive days and animals in the fourth group were treated with buspirone for 14 consecutive days, and 30 minutes after the last buspirone administration were administered with P400 (BUSP + P400). Results: No toxicity signs or death were observed in buspirone-treated animals. P400 group showed a significant increase in nitrite production and lipid peroxidation after seizures. Moreover, reduction in both the lipid peroxidation level (65\%) and nitrite content (85\%) as well as an increase in superoxide dismutase activity was detected following P400 injection in the hippocampus of buspirone-pretreated mice. Discussion: Pretreatment with BUSP increased latency to first seizure, decreased the mortality rate and number of animals that presented seizures and progression to status epilepticus, showing potent anticonvulsant effects associated with reduction of hippocampal oxidative stress.
\end{abstract}

Ferreira PB, et al. / Rev Psiq Clín. 2012;39(5):153-6

Keywords: Buspirone, seizures, oxidative stress, hippocampus, pilocarpine.

\section{Introdução}

A epilepsia do lobo temporal é uma doença crônica que pode interferir em inúmeros aspectos cognitivos, bem como pode ser frequentemente associada a um fator precipitante1. É um dos distúrbios neurológicos mais comuns e apresenta taxa de prevalência de $5 \% 2$.

A buspirona é um agonista parcial do receptor serotoninérgico do subtipo $5-\mathrm{HT}_{1 \mathrm{~A}}$ que pode modificar a concentração cerebral de serotonina (5-HT). Atualmente, a buspirona é utilizada no tratamento da ansiedade. Entretanto, estudos demonstram que pode haver alterações nas funções do sistema serotoninérgico durante a patogênese da epilepsia ${ }^{3,4}$, despertando, assim, o interesse na investigação da buspirona em modelos de epilepsia.

Nesse contexto, é sabido que as crises epilépticas induzidas por pilocarpina produzem alterações histopatológicas em muitas regiões cerebrais que podem ser associadas e/ou decorrentes da produção excessiva de radicais livres ${ }^{5}$. Alterações comportamentais durante as crises epilépticas em camundongos têm sido amplamente estudadas após a administração aguda de compostos antiepilépticos e/ou antioxidantes ${ }^{6}$. Durante as crises epilépticas induzidas por pilocarpina pode ser observada uma diminuição na concentração cerebral de 5-HT em roedores. A diminuição do conteúdo de 5-HT bem como o aumento do seu metabólito (ácido 5-hidroxiindolacético) podem ser fatores patogênicos relacionados com o estresse oxidativo cerebral7.

A neurotoxicidade observada durante as crises epilépticas pode ser associada ao estresse oxidativo. Considerando que há o envolvimento do receptor $5-\mathrm{HT}_{1 \mathrm{~A}}$ na fisiopatologia das crises epilépticas, pode ser sugerido que compostos que atuam nesse receptor podem exercer efeitos benéficos contra essas crises. Além disso, não é conhecido o papel das drogas serotoninérgicas sobre as defesas antioxidantes, bem como qual a influência desses compostos sobre o estresse oxidativo causado pelas crises epilépticas induzidas por 
pilocarpinas. No entanto, o estresse oxidativo pode ser associado ao aumento do conteúdo de 5-HT hipocampal em camundongos durante o estabelecimento das crises epilépticas e do estado de mal epiléptico induzidos por pilocarpina9.

Dessa forma, o objetivo do presente estudo foi investigar as alterações comportamentais e o efeito antioxidante da buspirona no hipocampo de camundongos no modelo de epilepsia induzida por pilocarpina.

\section{Métodos}

Foram utilizados camundongos Swiss adultos machos com 2 meses de idade, com peso variando de 25-30 g, provenientes do Biotério Central do Centro de Ciências Agrárias da Universidade Federal do Piauí (UFPI). Durante todos os experimentos, os animais foram mantidos em gaiolas de acrílico de $30 \times 30 \mathrm{~cm}^{2} \mathrm{com}$ no máximo seis animais. Os camundongos foram mantidos em uma sala com temperatura controlada de $25 \pm 1{ }^{\circ} \mathrm{C}$, com ciclo claro/escuro alternado de 12 horas, recebendo ração padrão tipo Purina e água ad libitum. Todos os experimentos foram aprovados pelo Comitê de Ética em Experimentação com Animais da UFPI (número de aprovação: 0021/10).

Durante os experimentos, os 48 animais utilizados no estudo foram divididos em quatro grupos. O primeiro e o segundo grupo foram tratados diariamente por via oral com buspirona $(5 \mathrm{mg} / \mathrm{kg}, \mathrm{n}$ $=12$ ) ou com solução salina $0,9 \%(\mathrm{n}=12)$. Após $30 \mathrm{~min}$ da administração da última dose da buspirona ou solução salina, os animais receberam cloridrato de pilocarpina na dose de $400 \mathrm{mg} / \mathrm{kg}$ por via intraperitoneal $(\mathrm{n}=12)$. Os camundongos desses dois grupos foram observados durante $1 \mathrm{~h}$ para registrar as alterações comportamentais. Os tratamentos descritos anteriormente representam os grupos BUSP + P400 e P400, respectivamente.

Por sua vez, o terceiro e o quarto grupo receberam diariamente por via oral solução salina $0,9 \%(n=12)$ ou buspirona $(5 \mathrm{mg} / \mathrm{kg}, \mathrm{n}=12)$. Da mesma forma, os camundongos desses grupos foram observados durante $1 \mathrm{~h}$ para registrar as alterações comportamentais. Os tratamentos descritos representam os grupos controle e BUSP, respectivamente.

Durante $1 \mathrm{~h}$ após os tratamentos, os animais foram observados para verificar a presença de sinais colinérgicos periféricos (SCP), tremores, movimentos estereotipados, crises epilépticas, estado de mal epiléptico e a taxa de mortalidade em cada grupo experimental de acordo com método previamente descrito por Freitas et al.7. Além disso, foi determinada a latência para o aparecimento da primeira crise epiléptica e do desenvolvimento do estado de mal epiléptico.

Após o período de observação, os animais que desenvolveram crises epilépticas e estado de mal epiléptico e sobreviveram ao período de observação foram eutanasiados. Em seguida, seus cérebros foram removidos para dissecação sobre gelo do hipocampo de ambos os lados do cérebro. O hipocampo foi utilizado para preparar o homogenato a $10 \%(\mathrm{p} / \mathrm{v})$ em tampão fosfato $(\mathrm{pH} 7,4)$ para a realização do estudo dos efeitos antioxidantes da buspirona no modelo de epilepsia induzida por pilocarpina.

O grau de peroxidação lipídica foi medido por meio da determinação do conteúdo de substâncias reativas com o ácido tiobarbitúrico (TBARS), conforme o método de Draper e Hadley ${ }^{10}$. Os resultados foram expressos em nanomol de MDA/g de tecido. Para determinar o conteúdo de nitrito, foi feita uma equação da reta para o cálculo das concentrações do teste de Green et al. ${ }^{11}$. As leituras foram realizadas a $560 \mathrm{~nm}$ e os resultados, expressos em mM.

Para determinação da atividade da catalase, foi utilizada a reação que tem como princípio básico a medida da velocidade de produção de $\mathrm{O}_{2}$ e $\mathrm{H}_{2} \mathrm{O}$ à proporção que o $\mathrm{H}_{2} \mathrm{O}_{2}$ é degradado, de acordo com o método previamente descrito por Maehly e Chance ${ }^{12}$ e Chance e Maehly ${ }^{13}$. A atividade dessa enzima foi medida em $230 \mathrm{~nm}$, por meio de um espectrofotômetro pela variação da absorbância por minuto, durante 6 minutos, e os resultados foram expressos em U/ $\mu \mathrm{g}$ de proteína.

A atividade da superóxido dismutase (SOD) foi determinada por meio da técnica previamente descrita anteriormente por Flohé e Otting ${ }^{14}$. A atividade da SOD das amostras foi calculada usando a média das absorções lineares obtidas durante 6 min pela curva a 550 $\mathrm{nm}$. Os resultados foram expressos em U/mg de proteína. Para todos os testes antioxidantes, a concentração da proteína nos homogenatos foi obtida por meio do método descrito por Lowry et al. ${ }^{15}$.

Para análise estatística dos resultados que obedeciam a uma distribuição paramétrica, foram utilizados a Análise de Variância (ANOVA) para múltiplas comparações e o teste de $t$-Student Newman Keuls como post hoc, teste pelo programa GraphPad Prism versão 3.00 para Windows, GraphPad Software, San Diego California USA. Copyright (c) 1994-1999 por GraphPad software. Os dados não paramétricos (porcentagens) foram analisados pelo mesmo programa, utilizando o teste do qui-quadrado. As diferenças foram consideradas estatisticamente significativas a partir de $\mathrm{p}<0,05$.

\section{Resultados}

Durante a primeira hora de observação, todos os camundongos do grupo P400 apresentaram sinais colinérgicos periféricos (SCP), como miose, piloereção, cromodacriorreia, salivação, diarreia, diurese e também movimentos estereotipados (ME), envolvendo o aumento da atividade de roer, coçar, mastigar e wet-dog shakes.

Todos os animais tratados por via intraperitoneal com pilocarpina na dose de $400 \mathrm{mg} / \mathrm{kg}$ (grupo P400) apresentaram crises epilépticas que progrediram para o estado de mal epiléptico (EME), e o aparecimento da primeira crise epiléptica ocorreu em 7,97 \pm 1,69 min.

Por sua vez, todos os animais pré-tratados com buspirona selecionados para este estudo foram observados durante $24 \mathrm{~h}$ após a administração de pilocarpina e manifestaram uma diminuição de 27\%, 45\% e 45\% no índice de tremores, crises epilépticas e EME (p $<0,001$ ), respectivamente (Tabela 1). Por outro lado, os parâmetros comportamentais relacionados aos SCP e ME foram observados de forma semelhante ao grupo P400 ( $p>0,05)$.

Tabela 1. Efeitos do pré-tratamento com buspirona nas alterações comportamentais durante as crises epilépticas induzidas por pilocarpina

\begin{tabular}{|l|c|c|c|c|c|}
\hline Grupos & $\begin{array}{c}\text { SCP } \\
(\%)\end{array}$ & $\begin{array}{c}\text { ME } \\
(\%)\end{array}$ & $\begin{array}{c}\text { Tremores } \\
(\%)\end{array}$ & $\begin{array}{c}\text { Crises } \\
\text { epilépticas } \\
(\%)\end{array}$ & $\begin{array}{c}\text { EME } \\
(\%)\end{array}$ \\
\hline P400 & 100 & 100 & 100 & 100 & 100 \\
\hline BUSP + P400 & 100 & 100 & $73 a$ & $55 a$ & $55 a$ \\
\hline BUSP & 00 & 00 & 00 & 00 & 00 \\
\hline
\end{tabular}

a p < 0,001, quando comparado ao grupo P400 (teste do qui-quadrado). SCP: sinais colinérgicos periféricos; ME: movimentos estereotipados; EME: estado de mal epiléptico.

Os resultados do estudo demonstram que durante o período do tratamento não foi observado nenhum sinal de toxicidade, bem como não foram detectadas alterações comportamentais e nenhuma morte entre os animais tratados apenas com buspirona (grupo BUSP). Em relação à taxa de mortalidade entre os animais observados em cada grupo experimental, foi vista uma taxa de $46 \%$ e $100 \%$ nos grupos BUSP + P400 e P400, respectivamente (Tabela 2).

Tabela 2. Efeito do pré-tratamento com buspirona nas latências para 0 aparecimento da primeira crise epiléptica (APCE) e do estado de mal epiléptico (LIEME) durante as crises epilépticas induzidas por pilocarpina e taxa de mortalidade em camundongos adultos

\begin{tabular}{|l|c|c|c|}
\hline Grupos & $\begin{array}{c}\text { APCE } \\
(\mathbf{m i n})\end{array}$ & $\begin{array}{c}\text { LIEME } \\
(\mathrm{min})\end{array}$ & $\begin{array}{c}\text { Taxa de } \\
\text { mortalidade } \\
(\%)\end{array}$ \\
\hline P400 & $7,90 \pm 1,68$ & $14,95 \pm 1,57$ & 100 \\
\hline BUSP + P400 & $17,4 \pm 1,53^{\mathrm{a}}$ & $24,17 \pm 3,49 \mathrm{a}$ & $54^{*}$ \\
\hline BUSP & 00 & 00 & 00 \\
\hline
\end{tabular}

As latências foram expressas como a média \pm EPM em minutos (min) do número de animais usados nos experimentos, enquanto a taxa de mortalidade foi expressa em porcentagem. a $p<$ 0,001 , quando comparado ao grupo P400 (ANOVA e teste $t$-Student-Neuman-Keuls como post hoc teste); * $\mathrm{p}<0,001$, quando comparado ao grupo P400 (teste do qui-quadrado). 
Além disso, foi verificado um aumento de $120 \%$ e $62 \%$ nas latências para aparecimento da primeira crise epiléptica (APCE) e do desenvolvimento do EME no grupo BUSP + P400 em comparação ao $\mathrm{P} 400$ ( $\mathrm{p}<0,001)$, respectivamente (Tabela 2 ).

De acordo com o observado na figura 1 , no grupo tratado com P400 houve um aumento significativo ( $\mathrm{p}<0,001)$ no nível de peroxidação lipídica (52\%) e no conteúdo de nitrito (53\%), bem como foi detectado um aumento de $76 \%$ na atividade da enzima catalase ( $\mathrm{p}<0,001)$ em comparação ao grupo controle. Por outro lado, não foram detectadas alterações significativas na atividade da superóxido dismutase no grupo P400 ( $\mathrm{p}>0,05)$. No hipocampo dos animais que receberam o pré-tratamento com buspirona e que 30 min após receberam $\mathrm{P} 400$, foi observada uma redução significativa do nível de peroxidação lipídica (35\%) e nitrito (85\%), bem como um aumento apenas da atividade da enzima superóxido dismutase (45\%) quando comparado com o grupo P400. Já na atividade da enzima catalase no grupo BUSP + P400 não foi vista nenhuma mudança em relação ao grupo $\mathrm{P} 400(\mathrm{p}>0,05)$.

Além disso, quando comparado o grupo BUSP ao controle, foi detectada uma redução no conteúdo de nitrito de $51 \%$ e de $57 \%$ no nível de peroxidação lipídica ( $\mathrm{p}<0,001)$, bem como pode ser observado um aumento de $25 \%$ e $55 \%$ das atividades das enzimas catalase e superóxido dismutase $(\mathrm{p}<0,001)$, respectivamente, sugerindo um efeito antioxidante para a buspirona.

\section{Discussão}

Estudos sugerem que o estresse oxidativo produzido durante o estabelecimento das crises epilépticas induzidas por pilocarpina possivelmente pode ocorrer por meio da produção excessiva de radicais livres ${ }^{16}$. O estresse oxidativo é um dos possíveis mecanismos responsáveis pelas crises epilépticas induzidas por pilocarpina, entre esses pode ser destacada a suscetibilidade cerebral ao processo oxidativo. $\mathrm{O}$ cérebro recebe grandes quantidades de $\mathrm{O}_{2}$, bem como apresenta elevado conteúdo de substratos para oxidação associada a uma baixa atividade das enzimas antioxidantes, sendo, portanto, extremamente suscetível aos danos produzidos pelo estresse oxidativo9,17.

A relação entre as enzimas antioxidantes e o seu papel na remoção dos radicais livres em determinadas áreas cerebrais pode ser sugerida no modelo de epilepsia induzida por pilocarpina, bem como pode ser observado que o aumento do conteúdo das espécies reativas derivadas do oxigênio pode estar relacionado com estabelecimento da neurodegeneração induzida pelas crises epilépticas ${ }^{18}$. As pesquisas atuais têm sugerido que os compostos antioxidantes podem induzir um papel protetor contra a neurotoxicidade observada durante as crises epilépticas em nível celular ${ }^{19-21}$.

Outro estudo sugere que um aumento na formação de radicais livres pode ser acompanhado de um aumento compensatório das atividades das enzimas antioxidantes. Além disso, o hipocampo durante as crises epilépticas pode ser particularmente sensível ao estresse oxidativo por causa de seus altos níveis de 5-HT, que induzem a formação de radicais livres ${ }^{20}$, bem como pela diminuição da atividade das enzimas antioxidantes.

O presente estudo demonstrou que o pré-tratamento com buspirona pode produzir um aumento na atividade antioxidante das enzimas investigadas, bem como foi detectada uma redução na formação de radicais livres, sugerindo uma explicação parcial para o efeito antioxidante da buspirona. Além disso, houve diminuição no índice de crises epilépticas e menor taxa de mortalidade entre os animais pré-tratados com buspirona, sugerindo um papel dos radicais livres durante o processo de ocorrência e propagação dessas crises.

A produção de radicais lipídicos pode ser iniciada pela formação da peroxidação lipídica, que culmina na neurodegeneração observada em diferentes regiões cerebrais durante as crises epilépticas ${ }^{17}$. Neste estudo foi detectado um aumento na peroxidação lipídica hipocampal no grupo P400. Por outro lado, o tratamento prévio com buspirona bloqueou a peroxidação lipídica, sugerindo que a modulação do sistema serotoninérgico pode auxiliar na atividade antioxidante desse composto 22 . Nosso estudo concorda com os resultados obtidos por Ueda et al. ${ }^{23}$, que demonstraram um aumento na produção de radicais livres e peroxidação lipídica em neurônios após crises epilépticas.

Durante as convulsões, o nitrito e o nitrato têm sido implicados em muitos dos mecanismos moleculares do processo. Dessa forma, podem modular uma cascata de efeitos excitotóxicos no SNC e finalmente participar do subsequente dano neuronal e ativar outros mecanismos que potencializem os danos e a propagação do foco epiléptico ${ }^{22}$. Além disso, o pré-tratamento com buspirona
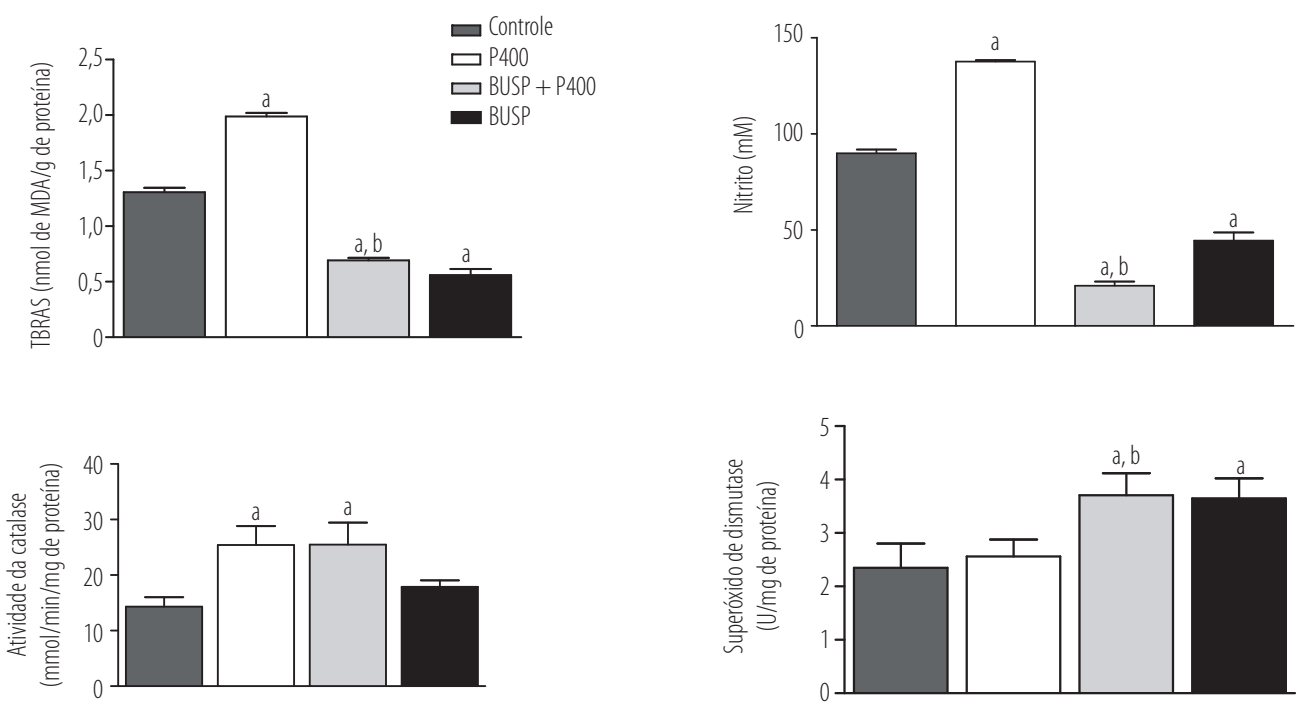

Figura 1. Nível de peroxidação lipídica e conteúdo de nitrito e atividade das enzimas catalase e superóxido dismutase (SOD) no hipocampo de camundongos pré-tratados com buspirona no modelo de epilepsia induzida por pilocarpina. Os resultados representam a média \pm EPM do número de animais usados nos experimentos. Para análise estatística, foram usados ANOVA e teste $t$-Student-Neuman-Keuls como post hoc teste. ap $<0,001$, quando comparado ao controle, e ${ }^{\mathrm{b}} \mathrm{p}<0,001$, quando comparado ao grupo P400 (ANOVA e teste $t$-Student-Neuman-Keuls como post hoc teste). 
diminuiu o conteúdo de nitrito no hipocampo demonstrando um efeito neuroprotetor que pode justificar a sua utilização como droga anticonvulsivante.

Além de investigar os efeitos sobre os radicais livres produzidos durante as crises epilépticas, neste estudo foi identificado o efeito da buspirona sobre a atividade da catalase e superóxido dismutase. A catalase é uma hemeproteína citoplasmática que catalisa a redução do peróxido de hidrogênio, produzindo um importante efeito antioxidante no organismo ${ }^{23}$. Os resultados do presente estudo não demonstraram modificações na atividade da enzima catalase no grupo pré-tratado com buspirona em relação ao grupo P400, sugerindo que essa enzima pode não estar envolvida no efeito antioxidante da buspirona no modelo estudado.

Durante o funcionamento fisiológico cerebral, mudanças na atividade hipocampal são acompanhadas por alterações no metabolismo cerebral induzindo modificações no fluxo sanguíneo dessa área e prejudicando seu funcionamento. Evidências clínicas e experimentais demonstraram possíveis alterações no nível basal do oxigênio durante e após as crises epilépticas, que podem ser demonstradas pela redução da concentração do oxigênio, bem como pelo elevado fluxo sanguíneo, provavelmente devido a uma vasodilatação secundária ${ }^{24}$.

Durante o estresse oxidativo são produzidos radicais livres como superóxido e radical hidroxila. Esses radicais têm sido implicados em uma variedade de processos neurodegenerativos, incluindo a epilepsia. Em todos esses eventos patológicos ocorrem inúmeras alterações bioquímicas que podem induzir mudanças na modulação das isoformas da enzima superóxido dismutase ${ }^{17,25-27}$. Dentre as enzimas antioxidantes, acredita-se que no hipocampo a principal é a SOD, já que essa área cerebral contém grande quantidade de zinco e cobre ${ }^{28-30}$. Nos animais do grupo BUSP + P400 e no BUSP, foi observado um aumento da atividade dessa enzima em comparação ao grupo P400, sugerindo que o efeito antioxidante exercido pela buspirona pode ser devido à modulação da atividade enzimática da SOD.

Os resultados demonstraram que o pré-tratamento com a buspirona aumentou a latência para o aparecimento da primeira crise epiléptica e diminuiu a taxa de mortalidade e o número de animais que apresentam crises epilépticas e que progridem para o estado de mal epiléptico. Com base nos resultados obtidos, pode-se sugerir que a buspirona apresenta efeito anticonvulsivante contra as crises epilépticas induzidas por pilocarpina, cujos mecanismos incluem a modulação na atividade de enzimas antioxidantes cerebrais, principalmente da superóxido dismutase. No entanto, futuros estudos devem ser realizados para melhor esclarecer o mecanismo de ação da buspirona, bem como os seus efeitos adversos decorrentes de um tratamento crônico.

\section{Referências}

1. Dodrill CB. Neuropsychological effects of seizures. Epilepsy Behav. 2004;5:S21-4.

2. De Lorenzo RJ, Raza M, Pal S, Rafiq A. Long-term alteration of calcium homeostastic mechanisms in the pilocarpine model of temporal lobe epilepsy. Brain Res. 2001;903:1-12.

3. Ohlsen RI, Pilowsky LS. The place of partial agonism in psychiatry: recent developments. J Psychopharmacol. 2005;19:408-13.

4. Watanabe A, Tohyama Y, Nguyen KQ, Hasegawa S, Debonnel G, Diksic $\mathrm{M}$. Regional brain serotonin synthesis is increased in the olfactory bulbectomy rat model of depression: an autoradiographic study. J Neurochem. 2003;85:469-75.

5. Freitas RM, Vasconcelos SMM, Souza FCF, Viana GSB, Fonteles MMF. Oxidative stress in the hippocampus after status epilepticus in rats. FEBS J. 2005;272:1307-12.

6. Militão GCG, Ferreira PMP, Freitas RM. Effects of lipoic acid on oxidative stress in rat striatum after pilocarpine-induced seizures. Neurochem Int. 2009;56:16-20.

7. Freitas RM, Viana GSB, Fonteles MMF. Níveis dos neurotransmissores estriatais durante o estado epiléptico. Rev Psiq Clín. 2003;30:76-9.
8. Freitas RLM, Santos IMS, Souza GF, Tomé AR, Saldanha GB, Freitas RM. Oxidative stress in rat hippocampus caused by pilocarpine-induced seizures is reversed by buspirone. Brain Res Bull. 2010;81:505-9.

9. Caccia S, Conti I, Vigano G, Garattini S. 1-(2-Pyrimidinyl)-piperazine as active metabolite of buspirone in man and rat. Pharmacology. 1986;33:46-51.

10. Draper HH, Hadley M. Malondialdehyde determination as an index of lipid peroxidation. Methods Enzymol. 1990;186:421-31.

11. Green LC, Tannenbaum SR, Goldman P. Nitrate synthesis in the germfree and conventional rat. Science. 1981;212:56-8.

12. Maehly AC, Chance B. The assay of catalases and peroxidases. Methods Biochem Anal. 1954;1:357-9.

13. Chance B, Maehly AC. Assay of catalases and peroxidases. Methods Enzymol. 1955;2:764-8.

14. Flohé L, Otting F. Superoxide dismutase assays. Meth Enzymol. 1984;105:93-104.

15. Lowry H, Rosebrough NJ, Farr AL, Randall RJ. Protein measurements with the folin phenol reagent. J Biol Chem. 1951;193:265-75.

16. Barros DO, Xavier SM, Barbosa CO, Silva RF, Maia FD, Oliveira AA, et al. Effects of the vitamin $\mathrm{E}$ in catalase activities in hippocampus after status epilepticus induced by pilocarpine in Wistar rats. Neurosci Lett. 2007;416:227-30.

17. Freitas RM, Sousa FCF, Vasconcelos SMM, Viana GSB, Fonteles MMF. Pilocarpine-induced seizures in adult rats: lipid peroxidation level, nitrite formation, GABAergic and glutamatergic receptor alterations in the hippocampus, striatum and frontal cortex. Pharmacol Biochem Behav. 2004;78:327-32.

18. Turski WA, Cavalheiro EA, Schwartz M, Czuczwarn SJ, Kleinrok Z, Turski L. Limbic seizures produced by pilocarpine in rats: a behavioural, electroencephalographic and neuropathological study. Behav Brain Res. 1983;9:315-35.

19. Tomé AR, Ferreira PMP, Freitas RM. The effects of alpha-tocopherol on hippocampal oxidative stress prior to in pilocarpine-induced seizures. Neurochem Res. 2010;35:580-7.

20. Halliwell B, Gutteridge JMC. Lipid peroxidation: a radical chain reaction. In: Halliwell B, Gutteridge JMC, editors. Free Radicals in Biology and Medicine. Oxford: Clarendon Press; 1996;188-266.

21. Santos PS, Feitosa CM, Saldanha GB, Tomé AR, Feng D, Freitas RM. Lipoic acid inhibits caspase-dependent and -independent cell death pathways and is neuroprotective against hippocampal damage after pilocarpine-induced seizures. Pharmacol Biochem Behav. 2011;97:531-7.

22. Gaspar P, Cases O, Maroteaux L. The developmental role of serotonin: news from mouse molecular genetics. Nat Rev Neurosci. 2003;4:1002-12.

23. Ueda Y, Yokayama H, Niwa R, Konaka R, Ohya-Nishiguchi H, Kamada H. Generation of lipid radicals in hippocampal extracellular space during kainic acid-induced seizures in rats. Epilepsy Res. 1997;26:329-33.

24. Sosunov AA, Kruglyakov PP, McCann GM, Kasperser K, Khovryakiv AV, Ivanov NM, et al. Studies of damage to hippocampal neurons in inbred mouse lines in models of epilepsy using kainic acid and pilocarpine. Neurosci Behav Physiol. 2005;35:623-38.

25. Freitas RM. Vigabatrina aumenta atividade da superóxido dismutase no corpo estriado de ratos após crises convulsivas induzidas pela pilocarpina. Rev Psiq Clín. 2010;37:36-40.

26. Pong K, Yongqi Y, Doctrow SR, Baudry M. Attenuation zinc-induced intracellular dysfunction and neurotoxicity by a synthetic superoxide dismutase/catalase mimetic, in cultured cortical neurons. Brain Res. 2002;950:218-30.

27. Dymond AM, Crandall PH. Oxygen availability and blood flow in the temporal lobes during spontaneous epileptic seizures in men. Brain Res. 1976;102:191-6.

28. Naffah-Mazzacoratti MG, Cavalheiro EA, Ferreira EC, Abdalla DSP, Amado D, Bellissimo MI. Superoxide dismutase, glutathione peroxidase activities and the hydroperoxide concentration are modified in the hippocampus of epileptic rats. Epilepsy Res. 2001;46:121-8.

29. Danscher G, Fjerdingstad FJ, Fjerdingstad E, Fredems K. Heavy metal content in subdivisions of the rat hippocampus (zinc, lead and copper). Brain Res. 1976;112:442-6.

30. Murakami K, Kondo T, Epstein CJ, Chan PH. Overexpression of CuZnsuperoxide dismutase reduces hippocampal injury after global ischemia in transgenic mice. Stoke. 1997;28:1797-804. 